\title{
ON THE EXISTENCE OF IDEMPOTENT LIFTINGS
}

\author{
S. GREKAS
}

(Communicated by R. Danial Mauldin)

\begin{abstract}
An existence theorem for idempotent liftings is proved. This implies that every compact measure space with full support and separable measure algebra admits an idempotent lifting.
\end{abstract}

\section{INTRODUCTION AND REVIEW OF TERMINOLOGY}

The problems treated in this note derive from the viewpoint of lifting theory developed in the paper of P. Georgiou [5]. More precisely, we are concerned with the existence of idempotent liftings for a compact measure space.

The classical bibliography yields many positive results on the strong lifting property and Losert's example [9] shows that the existence of strong liftings is not always possible. However (except the known results concerning strong liftings), relatively little is known about the existence of idempotent liftings.

In this paper, using classical arguments of disintegration theory, we establish an existence theorem for idempotent liftings. As a consequence, we have that-without any set theoretical assumptions-a separable measure space admits always an idempotent lifting. We note, in passing, that Martin's Axiom (and the continuum hypothesis) implies also the strong lifting property (cf. [3, 11(b), p. 8]) but-in ZFC alone-the existence of a strong lifting, for every compact separable measure space, might be an indecidable problem, for all that is known.

1.1. Let $T$ be a compact space, $\mu$ a positive Radon measure on $T$ with supp $\mu=T, M^{\infty}(T, \mu)$ the algebra of bounded measurable real valued functions on $T, L^{\infty}(T, \mu)$ the Banach algebra $M^{\infty}(T, \mu)$ modulo negligible functions and $h \rightarrow \tilde{h}$ the canonical projection of $M^{\infty}(T, \mu)$ onto $L^{\infty}(T, \mu)$. For a lifting $r$ of $M^{\infty}(T, \mu)$ the function $\psi_{r}: T \rightarrow T$ is defined by: $r f=f \circ \psi_{r}$, $f \in C(T)$ (cf. [5]).

1.2. Let $\Lambda(T, \mu)$ be the set of all liftings for $(T, \mu)$. We define the operation $*: \Lambda(T, \mu) \times \Lambda(T, \mu) \rightarrow \Lambda(T, \mu):\left(r_{1}, r_{2}\right) \rightarrow r_{1} * r_{2}:\left(r_{1} * r_{2}\right) f:=\left(r_{1} f\right) \circ \psi_{r_{2}}$,

Received by the editors October 19, 1988.

1980 Mathematics Subject Classification (1985 Revision). Primary 28C15.

(c) 1989 American Mathematical Society $0002-9939 / 89 \$ 1.00+\$ .25$ per page 
$f \in M^{\infty}(T, \mu)$. Then, $(\Lambda(T, \mu), *)$ becomes a semigroup whose right unit elements are exactly the strong liftings for $(T, \mu)$ (cf. [5]).

A lifting $r$ for $(T, \mu)$ is called idempotent, if $r * r=r$. Clearly, $r$ is idempotent iff $r f=(r f) \circ \psi_{r}$ for all $f \in M^{\infty}(T, \mu)$.

1.3. Let $X, T$ be compact spaces, $\lambda, \mu$ positive Radon measures on $X, T$ resp. and $p: X \rightarrow T$ a $\lambda$-proper mapping, with $p(\lambda)=\mu$. Then, a family $\left(\nu_{t}\right)_{t \in T}$ of measures on $X$ will be said to constitute a $p$-disintegration of $\lambda[8]$, if for every function $f \geq 0$ on $X$ measurable, the following conditions are satisfied:

(1) $f$ is $\nu_{t}$-measurable a.e. $(\mu)$;

(2) the ( $\mu$-almost everywhere defined) function $t \rightarrow \nu_{t}(f)$ is $\mu$-measurable and for every subset $A$ of $T \mu$-measurable, we have

$$
\int_{p^{-1} A} f d \lambda=\int_{A} \nu_{t}(f) d \mu(t) \text {. }
$$

\section{THE LIFTING THEOREM}

Throughout this section $X, T$ are compact spaces, $\lambda, \mu$ positive Radon measures supported on $X, T$ resp. and $p: X \rightarrow T$ a $\lambda$-proper surjection with $p(\lambda)=\mu$.

Denote by $p_{*}$ the mapping: $L^{\infty}(T, \mu) \rightarrow L^{\infty}(X, \lambda): \tilde{g} \rightarrow g \tilde{o} p$.

We start proving a slight extension of a theorem by A. and C. Ionescu Tulcea [8, Ch. IX, p. 138, Th. 3].

2.1. Lemma. Let $r$ be a lifting for $(T, \mu)$. Then, there is a p-disintegration $\left(\nu_{t}\right)_{t \in T}$ of $\lambda$ with the properties:

(i) $\sup \nu_{t} \subset p^{-1}\left\{\psi_{r}(t)\right\}$, a.e. $(\mu)$,

(ii) $\langle f, \nu\rangle \leq r\langle f, \nu\rangle, f \in C_{+}(X)$ (where for a disintegration $\left(\mu_{t}\right)_{t \in T}$ and $a$ universally measurable function $h$ on $X,\langle h, \mu\rangle$ is the function $\left.t \rightarrow \mu_{t}(h)\right)$.

Proof. This is similar to that of $[8$, Ch. IX, p. 154, Th. 5]. We sketch the steps of the proof.

I. For $f \in C(X)$ define the measure $\nu_{f}$ on $T$ by

$$
\nu_{f}(g):=\lambda(f(g \circ p)), \quad g \in C(T) .
$$

We immediately verify that $\nu_{f}$ is absolutely continuous with respect to $\mu$ and by Radon-Nikodym's theorem [2], we find a function $\kappa_{f} \in M^{\infty}(T, \mu)$ such that

$$
\nu_{f}(A)=\int_{A} \kappa_{f}(t) d \mu(t), \quad \text { for all } \mu \text {-measurable sets } A \text {. }
$$

For $t \in T$, define the measure $\kappa_{t}$ on $X: \kappa_{t}(f):=r \kappa_{f}(t), f \in C(X)$. 
II. We shall prove that $\left(\kappa_{t}\right)_{t \in T}$ is a $p$-disintegration of $\lambda$. We easily verify that

(3) holds for every nonnegative $\mu$-measurable function $g$ and so,

$$
\lambda\left(f \cdot \chi_{p^{-1} A}\right)=\int_{A} \kappa_{t}(f) d \mu(t)=\int \kappa_{t}(f) d\left(\chi_{A} \mu\right)(t)
$$

(where $\chi_{C}$ denotes the characteristic function of a set $C$ and $\chi_{A} \mu$ the measure on $\left.T:\left(\chi_{A} \mu\right)(g):=\mu\left(\chi_{A} g\right), g \in C(T)\right)$.

Applying now [8, Ch. III, p. 40, Th. 3], to the family of functions $\left(r \kappa_{f}\right)_{f \in C_{+}(X)}$, we show that (5) holds for $f$ lower semi-continuous and (by the regularity of $\lambda)$ for every $f \in M^{\infty}(X, \lambda)$. This means that $\left(\kappa_{t}\right)_{t \in T}$ is a $p$-disintegration of $\lambda$.

III. Since $p$ is $\lambda$-proper, there exists a disjoint sequence $\left\{K_{n}\right\}$ of compact sets in $X$, such that $\lambda\left(X-\bigcup_{n} K_{n}\right)=0$ and each $p_{n}=p \mid K_{n}: K_{n} \rightarrow T$ is continuous. For $n \in \mathbf{N}$ and $t \in T$ consider the measure $\nu_{n, t}$ on $X: \nu_{n, t}(f):=$ $r\left\langle f \chi_{K_{n}}, \kappa\right\rangle(t), f \in C(X)$. Then, since $\sum_{n} \nu_{t}(f) \leq\|f\|_{\infty}, f \in C(X), t \in T$, we (can) define on $X$ the measure $\nu_{t}:=\sum_{n} \nu_{n, t}$. that

We claim that $\left(\nu_{t}\right)_{t \in T}$ is the desired $p$-disintegration. For this, we verify

A. For every $f \in C_{+}(X), \nu_{t}(f)=\kappa_{t}(f)$, a.e. $(\mu)$. Let $A$ be any $\mu$ measurable set. Then, by [8, Ch. III, p. 40, Th. 3], we have that $\int_{A} \nu_{t}(f) d \mu(t)=$ $\int_{A} \sum_{n} r\left\langle f \chi_{K_{n}}, \kappa\right\rangle(t) d \mu(t)=\sum_{n} \int_{A} \kappa_{t}\left(f \chi_{K_{n}}\right) d \mu(t)=\sum_{n} \lambda\left(f \chi_{K_{n}} \chi_{p^{-1} A}\right)=$ $\lambda\left(f \chi_{p^{-1} A}\right)$ and using also [8, Ch. III, p. 40, Th. 3] that able.

B. $\lambda\left(f \chi_{p^{-1} A}\right)=\int_{A} \nu_{t}(f) d \mu(t)$, for every $f \in M^{\infty}(X, \lambda)$ and $A \mu$-measur-

It remains to check that (i) is true ((ii) is obvious). For this, it is sufficient to verify that

If $A$ is a compact subset of $X, g \in C(X)$ with $\left.g\right|_{A} c=0$ and $\psi_{r}(t) \notin p(A)$, then $\nu_{t}(g)=0$.

In fact, under these notations,

$$
\begin{aligned}
\nu_{n, t}(g) & =r\left\langle g \chi_{K_{n}}, \kappa\right\rangle(t) \leq\|g\|_{\infty} r\left\langle\chi_{A \cap K_{n}}, \kappa\right\rangle \\
& \leq\|g\|_{\infty} \chi_{r p\left(A \cap K_{n}\right)}(t) \quad\left(\text { because } \kappa_{t}\left(A \cap K_{n}\right) \leq \chi_{p\left(A \cap K_{n}\right)}(t) \text { a.e. }(\mu)\right) \\
& \leq\|g\|_{\infty} \chi_{\psi_{r}^{-1} p\left(A \cap K_{n}\right)}(t) \quad \text { (because } p\left(A \cap K_{n}\right) \text { is compact) } \\
& \leq\|g\|_{\infty} \chi_{\psi_{r}^{-1} p(A)}(t)
\end{aligned}
$$

and (\#) follows.

2.2. Theorem. Suppose that $p_{*}$ is onto $L^{\infty}(X, \lambda)$ and there is an idempotent lifting for $(T, \mu)$. Then there is an idempotent lifting for $(X, \lambda)$.

Proof. We shall use the notations in the proof of Lemma 2.1. Suppose that $r$ is idempotent.

Claim 1. For every $t \in T, \kappa_{t}$ is a Dirac measure. 
Since $\left(\kappa_{t}\right)_{t \in T}$ is a $p$-disintegration of $\lambda$ and $r\langle 1, \kappa\rangle=\langle 1, \kappa\rangle, \kappa_{t}$ is a probability measure, for all $t$. We show that each $\kappa_{t}$ is a multiplicative functional (on $C(X)$ ). Indeed, take $f_{i} \in C(X), i=1,2$. By assumption, there is $g_{i} \in M^{\infty}(T, \mu)$ such that $f_{i}=g_{i} \circ p$ a.e. $(\mu)$. Then, for every $\mu$-mesurable set $A, \int_{A} \kappa_{t}\left(f_{i}\right) d \mu(t)=\int_{p^{-1} A} f_{i} d \lambda=\int_{p^{-1} A}\left(g_{i} \circ p\right) d \lambda=\int_{A} g_{i} d \mu$, so,

$$
\begin{gathered}
\left\langle f_{i}, \kappa\right\rangle=g_{i} \quad \text { a.e. }(\mu) \text { (and similarly), } \\
\left\langle f_{1} f_{2}, \kappa\right\rangle=g_{1} g_{2} \quad \text { a.e. }(\mu) .
\end{gathered}
$$

Thus, $r\left\langle f_{1} f_{2}, \kappa\right\rangle=\left\langle f_{1} f_{2}, \kappa\right\rangle=r\left(g_{1} g_{2}\right)=r g_{1} r g_{2}$ and Claim 1 follows.

Now, let $q: T \rightarrow X$ be the mapping defined by: $\kappa_{t}=\delta_{q(t)}, t \in T$, where $\delta_{x}$ is the Dirac measure at $x \in X$. Since $\left(\kappa_{t}\right)_{t \in T}$ is a $p$-disintegration of $\lambda, q$ satisfies the condition:

$$
(q \circ p)^{-1} A \underset{\lambda}{\equiv} A, \quad \text { for all } \lambda \text {-measurable sets } A .
$$

Claim 2. $\mu$-almost for all $t \in T$, we have $p \circ q(t)=\psi_{r}(t)$.

Since $\nu_{t}(1)=1$ a.e. $(\mu)$ and $\nu_{t} \leq \delta_{q(t)}$ for all $t \in T, \mu$-almost for all $t$, $\kappa_{t}=\nu_{t}$ and Claim 2 follows combining Claim 1 and Lemma 2.1 .

Define now the lifting $u$ of $M^{\infty}(X, \lambda)$ by: $u h:=r(h \circ q) \circ p, h \in M^{\infty}(X, \lambda)$. $u$ is in fact a lifting: the main task is to show that $u h \equiv_{\lambda} h$. But, this is obvious, since, by $(+), r(h \circ q) \circ p \equiv_{\lambda} h \circ q \circ p \equiv_{\lambda} h$. Moreover, for $h \in C(X)$ and $x \in X$, $u h(x)=r(h \circ q) \circ p(x)=r\langle h, \kappa\rangle \circ p(x)=\langle h, \kappa\rangle \circ p(x)=\kappa_{p(x)}(h)=h \circ q \circ p(x)$. This means that $\psi_{u}=q \circ p$.

Consider the sets

$$
E:=\left\{t \in T: \psi_{r}(t)=p \circ q(t)\right\}
$$

and

$$
F:=\left\{x \in X: u h(x)=(u h) \circ \psi_{u}(x)\right\} .
$$

Since $\psi_{u}=q \circ p$, we deduce $(u h) \circ \psi_{u}(x)=u h(x)$, for all $h \in M^{\infty}(X, \lambda)$ and $x \in p^{-1} E$. Thus, $\lambda(F)=\lambda(X)$.

Define now the mapping $v: L^{\infty}(X, \lambda) \rightarrow M^{\infty}(X, \lambda)$ :

$$
\tilde{h} \rightarrow v \tilde{h}: v \tilde{h}(x):= \begin{cases}u \tilde{h}(x), & x \in F, \\ \phi_{x} \tilde{h}, & x \notin F,\end{cases}
$$

where $\phi_{x}$ is a character of $L^{\infty}(X, \lambda)$ such that $\phi_{x} \tilde{f}=f(x), f \in C(X)$ (cf. $[8, \mathrm{Ch} . \mathrm{V}])$. It is easily seen that $v$ is a lifting for $(X, \lambda)$. We claim that $v$ is in fact idempotent. (8) shows that

$$
\psi_{v}(x)= \begin{cases}\psi_{u}(x), & x \in F, \\ x, & x \notin F .\end{cases}
$$

Take any $h \in M^{\infty}(X, \lambda)$ and $x \in X$. Then

$$
(v h) \circ \psi_{v}(x)=\left\{\begin{array}{ll}
v h\left(\psi_{u}(x)\right), & x \in F, \\
v h(x), & x \notin F,
\end{array}=\left\{\begin{array}{ll}
u h\left(\psi_{u}(x)\right), & x \in F, \\
v h(x), & x \notin F,
\end{array}=v h(x) .\right.\right.
$$

So, $v$ is idempotent. 
2.3. Corollary. Let $(X, \lambda)$ be a compact (probability) measure space with $\sup \lambda=X$ and separable measure algebra. Then, there exists an idempotent lifting for $(X, \lambda)$.

Proof. W.1.o.g. we can take $\lambda$ to be nonatomic. Let $\mu$ be the Lebesgue measure on $[0,1]$. By [10], there is a measure preserving surjection $p: X \rightarrow[0,1]$. Since $[0,1]$ is compact and metrizable, $p$ is $\lambda$-proper (cf. [2, Ch. IV, p. 179, Th. 4]). On the other hand, $p_{*}$ is onto (cf. [2]) and there is a strong lifting for $\mu$. The conclusion follows from Theorem 2.2.

2.4. Remarks and Examples. (a) Let $\mu$ be the Lebesgue measure on $T=$ $[0,1],(X, \lambda)$ the hyperstonian space associated with $(T, \mu), p$ the canonical mapping: $X \rightarrow T$ and $r$ an arbitrary strong lifting for $(T, \mu)$. Then (under the notations in the proof of 2.2 , one can easily verify that) $v$ provides an example of an idempotent lifting, which is not almost strong. (However, it is not known if-in general-the existence of idempotent liftings implies the strong lifting property).

(b) The special case of Theorem 2.2, where $p$ is continuous and $(T, \mu)$ admits a strong lifting, has been proved by P. Georgiou [6], using the "special disintegration" (cf. [4]).

(c) Suppose that there is an idempotent lifting for $(X, \nu)$. Then, every measure (supported on $X$ and) absolutely continuous with respect to $\nu$ admits an idempotent lifting (this has been proved in [7] and extends a theorem of $\mathrm{K}$. Bichteler-cf. [1]).

\section{ACKNOWLEDGMENT}

The author owes gratitude to Prof. P. Geogiou for a stimulating discussion on the subject of this paper.

\section{REFERENCES}

1. K. Bichteler, An existence theorem for strong liftings, J. of Math Analysis and Appl. 33 (1) (1971), 20-22.

2. N. Bourbaki, Integration, ch. IV, V, Hermann, Paris, 1965.

3. D. H. Fremlin, On two theorems of Mokobodzski, note of 23/6/77.

4. P. Georgiou, Über eine spezielle Desintegration, Math. Ann. 197 (1972), 279-285.

5. __ A semigroup structure in the space of liftings, Math. Ann. 208 (1974), 195-202.

6. ___ On "idempotent" liftings, in Lecture Notes in Math., vol. 794, Springer-Verlag, Berlin, Heidelberg, New York 1980, 254-260.

7. S. Grekas, On idempotent liftings, Bull. of the Greek Math. Soc., to appear.

8. A. and C. Ionescu Tulcea, Topics in the theory of lifting, Springer-Verlag, Berlin, Heidelberg, New York, 1969.

9. V. Losert, A measure space without the strong lifting property, Math. Ann. 239 (1979), 119-128.

10. R. E. Zink, On the structure of measure spaces, Acta Math. 107 (1962), 53-71.

Oitis 12, T.K 10672, Athens, Greece 